Original Contribution

\title{
PATHO-MORPHOLOGICAL AND CLINICAL EXAMINATIONS IN EXPERIMENTAL INFECTION OF GUINEA FOWL (NUMIDA MELEAGRIS) WITH LPAIV H6N2
}

\author{
Iv. D. Ivanov", Iv. S. Zarkov*, K. Koev ${ }^{2}$ \\ ${ }^{1}$ Department of General and Clinical Pathology, Faculty of Veterinary Medicine, Trakia University, Stara \\ Zagora, Bulgaria \\ ${ }^{2}$ Department of Microbiology, Infectious and Parasitic Diseases, Trakia University, Faculty of Veterinary \\ Medicine, Stara Zagora, Bulgaria
}

\begin{abstract}
Eighteen 2-month-old guinea fowl (Numididae meleagris) birds were intravenously infected with $100 \mu \mathrm{L}$ H6N2 virus, while three formed a control group - non-infected. The birds were clinically examined daily throughout the entire experimental period and no clinical symptoms of disease were observed. On days 7 , 14 and 21, six infected and 1 control birds were slaughtered for pathological investigations. All visceral organs were macroscopically analysed and samples from lungs, heart, spleen, liver, kidneys, pancreas, thymus, bursa and duodenum were immediately removed and fixed in $10 \%$ buffered formalin for at least 2 days. Slices of $5 \mu \mathrm{m}$ thickness were prepared, embedded in paraffin and stained with haematoxylin and eosin $(\mathrm{H} / \mathrm{E})$ by standard procedures. The preparations were examined on Leitz light microscope. From the conducted pathoanatomical examinations, notable findings included the smaller size of the spleen, thymus, and the bursa of Fabricius in all examined birds, compared to the control group. Microscopically, however, as a constant find in all infected birds, we observed reactions of different type and extent within the lymphoid tissue of the central and peripheral immunocompetent organs, which could be summarised in two primary groups - lymphoid-proliferative and degenerative. The changes observed in the birds' euthanised 21 days p.i. were considerably more pronounced. Within the organs of the central immune system (thymus and spleen) lesions of the atrophic-degenerative type were found out. The organs of the peripheral immune system (the spleen and the entire mucosa-associated lymphoid tissue, including the respiratory tract, the alimentary tract with Peyer's patches, separate follicles and caecal tonsils) exhibited simultaneously atrophic regressive changes in the spleen and varying degrees of lymphoid activity in the other areas. In two of the euthanised 14 days p.i. and three 21 days p.i. birds, a lymphoid proliferation of the nodular type within the mucosa-associated lymphoid tissue of the lungs was discovered.
\end{abstract}

Key words: Avian influenza virus, H6N2 subtype, Numida meleagris, histology

\section{INTRODUCTION}

Guinea fowls (Numididae) are a bird family from the order Galliformes, which also includes chickens, turkeys, pheasants, partridges and other AIV-sensitive representatives. Guinea fowl are encountered in a wild state in Africa and domesticated - on a global scale (1). From the known 6 guinea fowl species, the common guinea fowl (Numida meleagris) is the most widely distributed.

*Correspondence to: IVAN ZARKOV, Department of Microbiology, Infectious and Parasitic Diseases, Trakia University, Faculty of Veterinary Medicine, 6000 Stara Zagora, Bulgaria, E-mail:

ivanzarkov@yahoo.com

180
Guinea fowl reared under domestic conditions are most commonly in close contact with other bird species. The first literature data for spontaneous infections of guinea fowl with AIV date back to 2000 (2), showing that they could be infected from other birds and from their part, is a source of infection for other birds. All data for infection of guinea fowl available so far are related to HPAIV and HPNAI.

The high extent of spread of HPAIV H5N1 among birds has led to establishing infection in guinea fowl as well (3). In Kenya, guinea fowl isolates are available in H5N1 outbreaks (4). In Italy, an emerging infection with HPAIV H7N7 has affected 7 bird species - chickens, turkeys, 
emus, goose, ducks, pelicans and guinea fowl (5). The most recent data justify the performance of a monitoring survey for the presence of AIV in birds reared in domestic conditions, including guinea fowl (6), and from some of them, a H9N2 strain was isolated.

In an experiment with HPAIV H5N1 in guinea fowl, all birds died within 2 to 5 days (7).

The extent and nature of the observed lesions after experimental infection with H6N2 were most expressed in ducklings, followed by turkey poults and chickens, and depending on the time of exposure to the virus, the predominant lesions were the ones on the $7^{\text {th }}$ and $14^{\text {th }}$ day after infection (8).

The macroscopically established lesions were predominantly of the viraemic and necrobiotic type. We observed ecchymoses or vibices within the epicardium, endocardium and myocardium; numerous petechial haemorrhages in all lobes of the thymus gland. On the $21^{\text {st }}$ day after infection, the lobes of the thymus gland were atrophic in chickens, while in the other birds there were analogous alterations in the spleen and the bursa of Fabricius. A constantly present sign was the hyperaemia of the liver, spleen, kidneys and lungs. In one of the infected chickens, numerous ecchymoses were found within the mucosa of the small intestines on the $14^{\text {th }}$ day after infection (9).

The histologically established lesions confirmed and complemented the macroscopic findings. In birds, on the $7^{\text {th }}-14^{\text {th }}$ day after infection, there was a perivascularly-positioned congestion and haemorrhages in the lungs; in the epicardium there were congestion, haemorrhages and parenchymal dystrophy in the myocardium, as well as focal round-cell inflammatory proliferates in ducks; in the kidneys there were inter- and intratubular haemorrhages and dystrophic degenerative alterations in the epithelia of the straight excretory ducts; in the livers of all birds, regardless of the time of exposure, we found congestion and parenchymal dystrophy, while in the ducks, apart from these lesions, there were also haemorrhages of varying intensity and severe haemorrhagic infarctions. Among the detritus mass of the infarctions there were distinguishable single drops of hyaline. In all birds there was a strong atrophy of the spleen's white pulp. On the $21^{\text {st }}$ day after infection, in turkeys, a focal interstitial roundcell proliferate and haemorrhages were found in the pancreas, whereas in ducklings there were
IVANOV IV.D.,et al.

numerous necroses of various sizes in the parenchyma of the pancreas, some of which were haemorrhagically infarcted. In separate excretory ducts, located next to the necroses, there was a cystic dilatation. In the macroscopically spotted haemorrhages within the small intestine of a chicken, massive haemorrhages were established histologically within the lymphatic patches of the mucosa. Haemorrhages were predominant within the parenchyma of the thymus gland. After the $21^{\text {st }}$ day of infection, a high-degree atrophy of the follicles of the bursa of Fabricius was found in all bird species $(8,9)$.

From literature data, no information about guinea fowl infection with LPAIV is available. After the acknowledged considerable occurrence of the H6N2 strain in Bulgaria, infection of guinea fowl is quite possible. Therefore, our aim was to investigate the possibility for infection of guinea fowl with a H6N2 AIV isolate and to follow out the periods of virus carriership and shedding and histological investigation.

\section{MATERIAL AND METHODS}

\section{VIRUS AND INOCULUM PREPARATION}

The low-pathogenic avian influenza A virus (LPAIV) of the H6N2 subtype obtained from a mallard duck (Anas plathyrynchos) was used at a titre of $10^{5} \mathrm{ELD}_{50} / 0.1 \mathrm{~mL}$ (ELD $_{50}$ mean embryo lethal doses causing a $50 \%$ death rate in inoculated chicken embryos). Allantoic fluid was collected after inoculation of LPAIV (H6N2 subtype) into the allantoic sac (100 $\mu \mathrm{L})$ of 5 to 9day old chicken embryos (CE). Embryos were observed daily for 120 hours (when all were dead). Allantoic fluid derived from them was explored by haemagglutination assay (HA). Samples with haemagglutin titres of 1:128 were stored at $-84^{\circ} \mathrm{C}$ until used in the experiment (10).

\section{BIRDS AND PROTOCOL DESIGN}

Eighteen 2 month-old birds were intravenously infected with $100 \mu \mathrm{L}$ allantoic fluid from infected chicken embryos (CE) with H6N2 virus. Three birds were inoculated the same way, with non-infected allantoic fluid (uninfected control group, $\mathrm{n}=3$ ). The 2 groups of infected and uninfected birds were kept separately in $4 \mathrm{x} 4 \mathrm{~m}$ rooms at $1.8 \mathrm{~m}$ feeding and watering front, $20^{\circ} \mathrm{C}$ and $70 \%$ humidity. No vaccine and antibiotic were administered to the birds.

On days 7, 14 and 21, six infected and 1 control birds were slaughtered for pathological investigations. All visceral organs were macroscopically analysed and samples from 
lungs, heart, spleen, liver, kidneys, pancreas, thymus, bursa and duodenum were immediately removed and fixed in $10 \%$ buffered formalin for at least 2 days. Slices of $5 \mu \mathrm{m}$ of thickness were prepared, embedded in paraffin and stained with haematoxylin and eosin $(\mathrm{H} / \mathrm{E})$ by standard procedures. The preparations were examined on Leitz light microscope.

\section{CLINICAL EXAMINATIONS}

Birds were clinically examined daily throughout the whole experimental period.

\section{RESULTS}

Throughout the entire study period, no clinical signs of illness were observed.

During the conducted gross anatomical examinations, notable findings included the smaller size of the spleen, thymus and bursa of Fabricius in all infected birds, compared to the control group. In the other organs, there were no macroscopic changes.

Microscopically, however, constant findings in all test birds were reactions of varying type and extent of expression within the lymphoid tissue of the central and peripheral immunocompetent organs, from two primary types - lymphoid-proliferative and degenerative. The changes were more intense in the birds euthanised 21 days p.i.

Within the organs of the central immune system (thymus and spleen) there were lesions of the atrophic degenerative type, affecting the primary structural units (the lobules building up the parenchyma). In all inoculated birds of both groups, the volume of the thymus lobes was considerably reduced, in the cortical as well as the core part. There was a slight lymphoid presence in the central part of the lobes (Figure 1). The core part of the lobe-like structures in the propria of the B. Fabricii mucosa was also slightly active (pale, "empty"), (Figure 2).

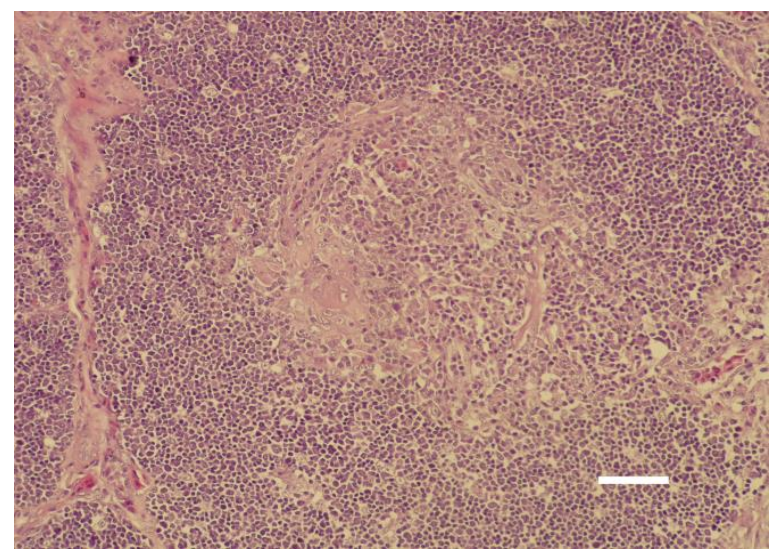

Figure 1. Cross-section, thymus gland lobe, guinea fowl 21 days p.i. Slight lymphoid presence and beginning of degenerative regenerative processes within the central zone, $\mathrm{H} / \mathrm{E}, \mathrm{Bar}=35 \mu \mathrm{M}$

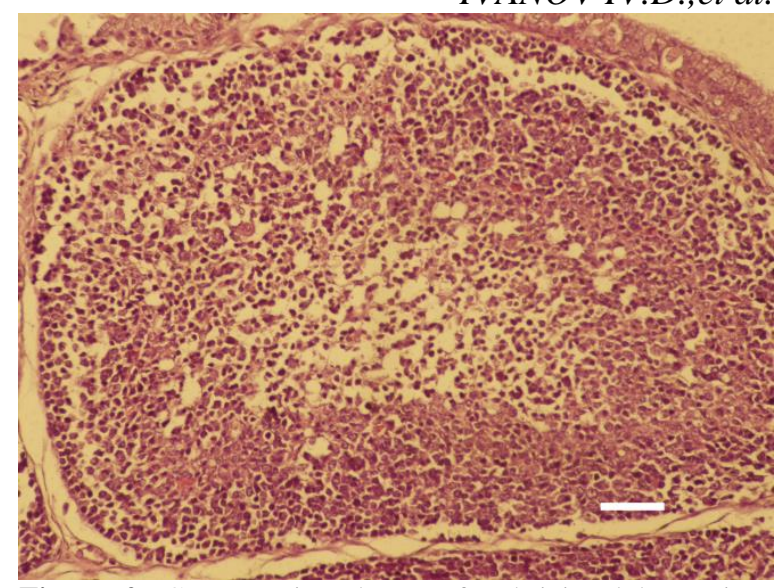

Figure 2. Cross-section, bursa of Fabricius lobe, guinea fowl 21 days p.i. Slightly active (pale, "empty") core part, $\mathrm{H} / \mathrm{E}, \mathrm{Bar}=25 \mu \mathrm{M}$

Within the organs of the peripheral immune system (the spleen and the entire mucosaassociated lymphoid tissue, including the respiratory tract, the alimentary tract with Peyer's patches, separate follicles and the caecal tonsils) there were simultaneous atrophicregressive changes in the spleen and varying degrees of increased lymphoid activity in the other areas of all birds from the experimental groups. Within the spleen, the periarteriolar lymphoid tissue in the T-dependent zone of the Malpighian bodies was atrophic. As a result of this, there was an aggregation of a large number (5-6 to 8-10) of central arterioles within a single observation field, even though sometimes lymphoid proliferation within the B-dependent zone was expressed (Figure 3).

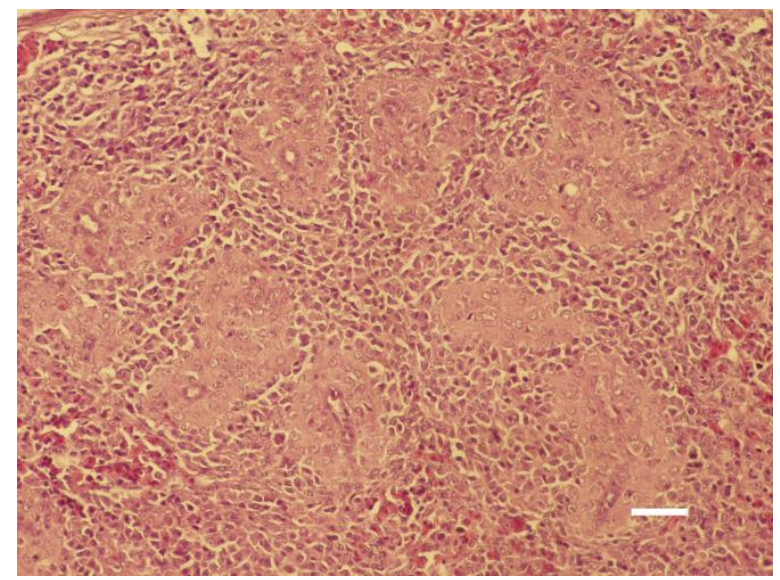

Figure 3. Spleen, guinea fowl, 21 days p.i. Accumulation of a large number of central arterioles within a single observation field, $\mathrm{H} / \mathrm{E}, \mathrm{Bar}=40 \mu \mathrm{M}$

The most significant lymphoid proliferation was observed in the different sections of the propria and the submucosa of the small intestines, with a predominant intensity in the middle and last third, and the caecal tonsils. The grown B- 
lymphoid cells around the central sinuses of the follicles led to various degrees of thickening of separate villi as well as of the mucosal membrane in some areas (Figure 4). A particularly developed mass was observed in the caecal tonsils, which formed indentations towards the intestinal lumen.

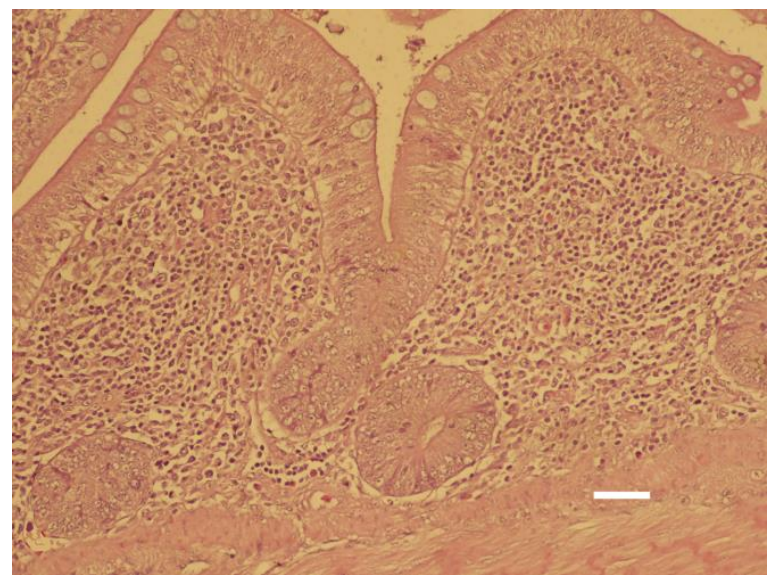

Figure 4. Cross section, small intestine, guinea fowl, 14 days p.i. Considerable lymphoid proliferation within the propria and the submucosa, leading to thickening of the intestinal villi and the mucosal membrane, $\mathrm{H} / \mathrm{E}, \mathrm{Bar}=30 \mu \mathrm{M}$

In two of the birds euthanised 14 days p.i. and in three euthanised 21 days p.i., there was lymphoid proliferation of the nodular type within the mucosa-associated lymphoid tissue of the lungs (Figure 5).

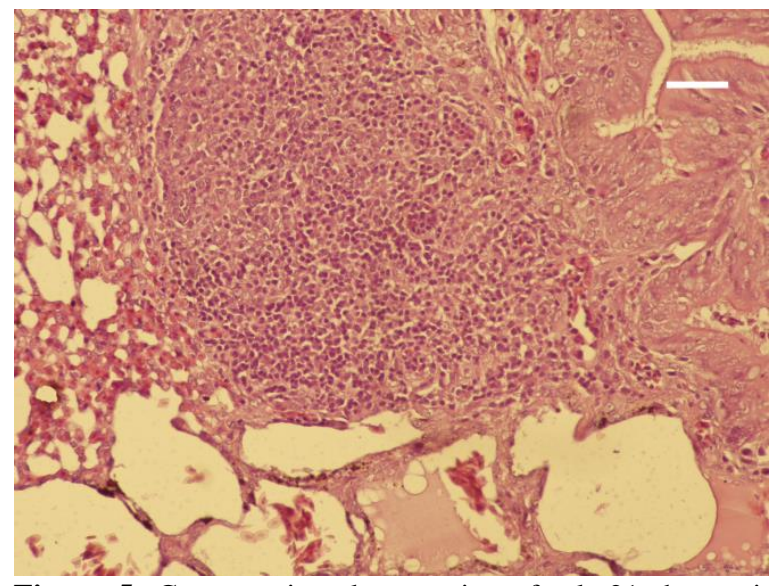

Figure 5. Cross-section, lung, guinea fowl, 21 days p.i. Interstitial pneumonia expressed through lymphoid proliferation of the nodular type, $\mathrm{H} / \mathrm{E}, \mathrm{Bar}=35 \mu \mathrm{M}$

Well-formed focal, single- multicentric, mononuclear round-cell proliferates (Figure 6 and 7) were observed primarily in the periportal spaces, but also outside them, within the liver and pancreas of two birds of the ones euthanised 14 days p.i. and three euthanised 21 days p.i. The newly grown cells caused compression atrophy of the surrounding parenchyma.

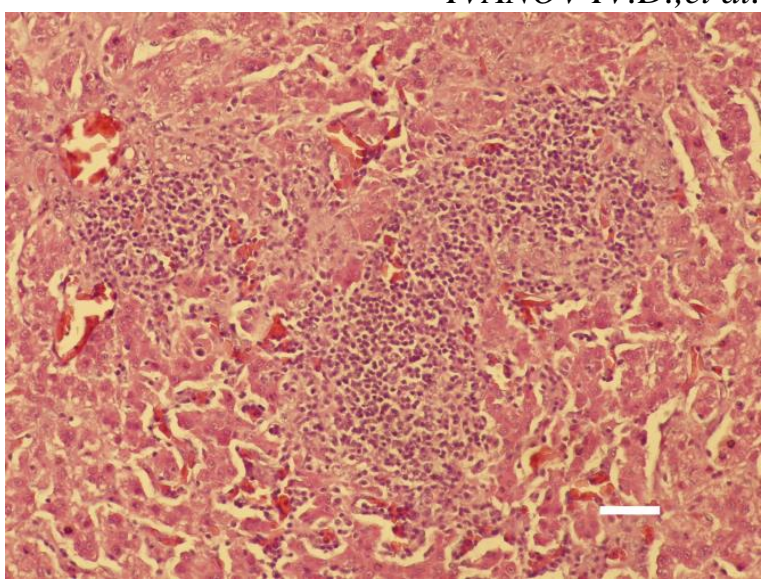

Figure 6. Cross-section, liver, guinea fowl, 14 days p.i. Multicentric, well-formed, mononuclear round-cell proliferates, $\mathrm{H} / \mathrm{E}, \mathrm{Bar}=40 \mu \mathrm{M}$

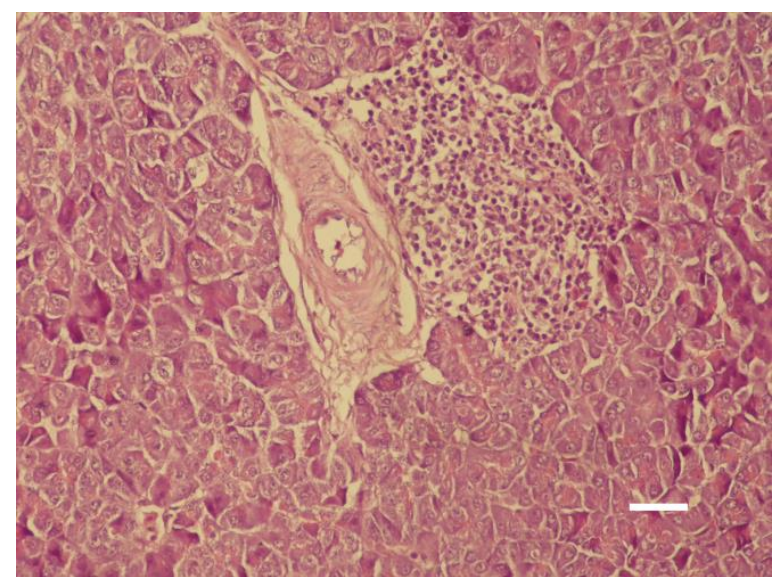

Figure 7. Cross-section, pancreas, guinea fowl, 21 days p.i. Well-formed mononuclear proliferate of the nodular type within the periportal spaces, $\mathrm{H} / \mathrm{E}, \mathrm{Bar}=30 \mu \mathrm{M}$

\section{DISCUSSION}

In the present studies, after a spot inoculation with LPAIV - H6N2, a reaction was observed only in the lymphoid tissue of the immunocompetent organs, unlike the changes of predominantly septicaemic nature in the other species of fowl (chickens, turkeys and ducks) inoculated with the same pathogen $(8,9)$. These differences could eventually be connected to the phylogenetic differences of the separate bird species. Even though the AI-specific clinical morphological signs of septicaemic nature were absent in the guinea fowl from this experiment, these birds could also be susceptible, carriers and spreaders of H6N2. Thus, even if we assume a host resistance of the guinea fowl towards LPAIV - H6N2, it apparently has a tropism towards lymphoid and immunocompetent tissue, which can be related to the respective immunosuppressive effect. Considering this, the results from our studies correspond to an extent with the findings of Kapczynski et al.,2013 (11), which report microscopically established tissue alterations of the apoptosis type and lymphoid 
depletion in the thymus and the bursa of Fabricius, spleen and caecal tonsils when chickens are infected with H7N3 - HPAIV. In the same case, similar to our findings yet differing in the severity of expression were the lymphohistiocytic hepatitis, interstitial pneumonia and the lesions within the pancreas.

\section{CONCLUSIONS}

The results from the study indicate that the guinea fowl infected with LPAIV subtype H6N2 did not exhibit any clinical signs of illness. Pathoanatomical changes were established (smaller sizes of the spleen, thymus and the bursa of Fabricius). The microscopic studies found reactions of various types and extent of expression within the lymphoid tissue of the central and peripheral immunocompetent organs, exhibited as lymphoid-proliferative and degenerative changes.

\section{REFERENCES}

1. Zheng, T., Adlam, B., Rawdon, T., Stanislawek, W., Corc, S., Hope, V., Buddle, B., Grimwood, K., Baker, M., O’Keefe, J. and Huang, Q. A cross-sectional survey of influenza A infection, and management practices in small rural backyard poultry flocks in two regions of New Zeland. New Zeland Veterinary Journal, 58: 2, 74-80, 2010.

2. Alexander, D.J., A review of avian influenza in different bird species. Veterinary Microbiology, 74: 3-13, 2000.

3. Kuiken, T., J. van den Brand, D. van Riel, M. Pantin-Jackwood, and D. E. Swayne. Comparative Pathology of Select Agent Influenza A Virus Infections, Veterinary Pathology , 47: 5, 893-914, 2010.

4. Fasina, F. O., Bisschop, S. P. R., Joannis T.M.,. Lombin, L.H and Abolnik, D. C. Molecular characterization and epidemiology of the highly pathogenic avian influenza
IVANOV IV.D.,et al.

$\mathrm{H} 5 \mathrm{~N} 1$ in Nigeria. Epidemiol. Infect., 137: 456-463, 2009.

5. Philippa, J. D. W., Munster, V., Bolhuis, H., Bestebroer, T., Schaftenaar, W., Beyer, W., Fouchier, R., Kuiken and Osterhaus, A. Higly pathogenic avian influenza (H7N7): Vaccination of zoo birds and transmission to non-poultry species. J. Vaccine, 23: 57435750, 2005.

6. Terregino, C., De Nardi, R., Guberti ,V., Scremin, M., Raffini, E., Martin, A. M., Cattoli, G., Bonfanti, L. and Capua, I. Active surveillance for avian influenza viruses in wild birds and backyard flocks in Northern Italy during 2004 to 2006, Avian Pathology, 36, 4: 337-344, 2007.

7. Perkins, L. E.\& Swayne, D. E. Pathogenicity of a Hong Kong - origin H5N1 Highly pathogenic avian influenza virus for emus ,geese, ducks, and pigeons. Avian Diseases, 46, 1: 53-63, 2002.

8. Zarkov I. S. \& Dinev, I. Clinical and morphological studies of ducklings experimentally infected with a H6N2 strain isolate of low pathogen avian influenza A virus. Revue Med. Vet., 163, 2, 64-69, 2012.

9. Zarkov, Iv. Studies on some biological properties of the Influenza A virus and characteristics of the infection in birds. Disertation DVM , Stara Zagora, 1-365, 2007

10.Anonymous. Version adopted by the World Assembly of Delegates of the OIE in May 2009, OIE Terrestrial Manual, Chapter 2.3.4., Avian Influenza, 1-20, 2009.

11.Kapczynski, D., Pantin-Jackwood, M., Guzman, S.G., Ricardez, Y., Spackman E., Bertran K., Suarez, D.L. and Swayne, D.E. Characterization of the Highly Pathogenic Avian Influenza H7N3 Virus Isolated from Poultry in an Outbreak in Mexico: Pathobiology and Vaccine Protection. Journal of Virology, 16, 9086-9089, 2013. 